\title{
Comparison of meat versus soya based high-protein diets on faecal microbiota and microbial metabolites
}

\author{
S.W. Gratz ${ }^{1}$, L. Scobbie ${ }^{1}$, A.J. Richardson ${ }^{1}$, X. Zhang ${ }_{1}^{2}$, C. Fyfe ${ }^{1}$, F.M. Farquharson ${ }^{1}$, \\ G. Duncan ${ }^{1}$, J. Filipe ${ }^{3}$, W.Y. Zhu ${ }^{2}$, A.M. Johnstone ${ }^{1}$, P. Louis ${ }^{1}$ and W.R. Russell ${ }^{1}$ \\ ${ }^{1}$ Rowett Institute, University of Aberdeen, Aberdeen, UK, \\ ${ }^{2}$ College of Animal Science and Technology, Nanjing Agricultural University, Nanjing, China and \\ ${ }^{3}$ Biomathematics and Statistics Scotland, Aberdeen, UK
}

High-protein, low carbohydrate diets are popular and efficient weight loss regimes, but we have previously shown that meat based high-protein diets had a negative effect on faecal metabolites and gut microbiota ${ }^{(1)}$. We therefore conducted a study to investigate whether vegetarian high-protein diets would have the same effect.

After 3 days on a balanced weight maintenance diet, 20 obese men received two high-protein weight loss diets with either beef or soya as the main protein source for 2 weeks each ${ }^{(2)}$. Faecal samples were collected at the end of each dietary period and faecal microbial DNA was extracted for qPCR bacterial profiling. Faecal water was extracted by high-speed centrifugation for short chain fatty acid (SCFA) analysis by GC and metabolite analysis by LC-MS/MS. Data were analysed by ANOVA with volunteer as a random effect and diet as a fixed effect and where diet effect was significant $(\mathrm{P}<0.05)$, means were compared with post hoc t-test.

The soya diet resulted in significant $(\mathrm{p}<0.05)$ shifts in gut microbiota composition increasing the proportions of Faecalibacterium prausnitzii and Lactobacillus spp. while decreasing the proportions of bacteria related to some Ruminococcus spp., Oscillibacter, Desulfovibrio spp. and Methanogens compared to the meat diet. Total faecal SCFA and the proportions of individual SCFA did not differ between individuals consuming meat or soya diets $(p>0.05$ ). Protein-derived metabolites (phenylacetic acid, phenylpyruvic acid and the proportion of branched-chain fatty acids iso-butyrate and iso-valerate) tended to be higher on both high-protein diets with no significant differences between soya and meat diets $(\mathrm{p}>0.05)$.

Several toxic and proliferative polyamines were significantly lower $(\mathrm{p}<0.05)$ on the soya diet compared to meat (cadaverine $69.4 \%$ decrease, piperidine $66.0 \%$ decrease, spermidine $49.4 \%$ decrease, spermine $81.5 \%$ decrease).

Heterocyclic amines were also significantly $(\mathrm{p}<0.05)$ lower on the soya diet compared to meat (IQ 26.7\% decrease, MeIQ $36.7 \%$ decrease, MeIQx 44.8\% decrease, 4,8DiMeIQx 69.6\% decrease, DMIP 30.9\% decrease). N-nitrosocompounds were also significantly lower on soya diet compared to meat $(41.1 \%$ decrease) whereas genotoxicity of faecal waters remained unaltered by diet changes $(\mathrm{p}=$ $0.56)$.

These results suggest that high-protein diets based on plant protein are less problematic in terms of intestinal health when compared to meat-based diets and might offer a safe option to support weight loss regimes.

1. Russell WR, Gratz SW, Duncan S, Holtrop G, et al. (2011) Am J Clin Nutr 93, 1062-1072.

2. Neacsu M, Fyfe C, Horgan G \& Johnstone AM (2014) Am J Clin Nutr 100, 548-558. 